\title{
n-3 polyunsaturated fatty acids abrogate mTORC1/2 signaling and inhibit adrenocortical carcinoma growth in vitro and in vivo
}

\author{
JUN LIU ${ }^{1,2^{*}}$, MEINIAN XU ${ }^{3 *}$, YONGBIN ZHAO ${ }^{1}$, CHUNPING AO $^{3}$, YUKUN WU $^{3}$, ZHENGUO CHEN $^{3}$, \\ BANGQI WANG ${ }^{1}, \mathrm{XIAOCHUN} \mathrm{BAI}^{3}, \mathrm{MING} \mathrm{LI}^{3}$ and WEILIE HU ${ }^{1}$ \\ ${ }^{1}$ Department of Urology, Guangzhou General Hospital of Guangzhou Military Command, Guangzhou 510010; \\ ${ }^{2}$ Guangzhou Huabo Bio-Pharmaceutical Research Institute, Guangzhou 510010; ${ }^{3}$ Department of Cell Biology, \\ School of Basic Medical Sciences, Southern Medical University, Guangzhou 510515, P.R. China
}

Received December 10, 2015; Accepted January 6, 2016

DOI: $10.3892 /$ or. 2016.4720

\begin{abstract}
PUFAs) are essential for human health and have been reported to reduce the risk of cancer, inhibit the growth of various types of tumors both in vitro and in vivo, and affect adrenal function. However, their effects on adrenocortical carcinoma (ACC) are not known. In the present study, we demonstrated that docosahexenoic acid (DHA) inhibited ACC cell proliferation, colony formation and cell cycle progression, and promoted apoptosis. In addition, ectopic expression of fat-1, a desaturase that converts n-6 to n-3 PUFAs endogenously, also inhibited ACC cell proliferation. Moreover, supplementing n-3 PUFAs in the diet efficiently prevented ACC cell growth in xenograft models. Notably, implanted ACC cells were unable to grow in fat-1 transgenic severe combined immune deficiency mice. Further study revealed that exogenous and endogenous n-3 PUFAs efficiently suppressed both mTOR complex 1 (mTORC1) and mTORC2 signaling in ACC in vitro and in vivo. Taken together, our findings provide comprehensive preclinical evidence that
\end{abstract}

Correspondence to: Professor Ming Li, Department of Cell Biology, School of Basic Medical Sciences, Southern Medical University, Guangzhou 510515, P.R. China

E-mail: looselm@126.com

Professor Weilie Hu, Department of Urology, Guangzhou General Hospital of Guangzhou Military Command, Guangzhou 510010, P.R. China

E-mail: huwl-mr@vip.sina.com

*Contributed equally

Abbreviations: ACC, adrenocortical carcinoma; n-3 PUFAs, $\omega-3$ polyunsaturated fatty acids; $n-6$ PUFAs, $\omega-6$ polyunsaturated fatty acids; mTOR, mammalian target of rapamycin; mTORC1, mTOR complex 1; mTORC2, mTOR complex 2; SCID, severe combined immune deficiency

Key words: adrenocortical carcinoma, n-3 polyunsaturated fatty acids, mammalian target of rapamycin n-3 PUFAs efficiently prevent ACC growth by inhibiting mTORC1/2, which may have important implications in the treatment of ACC.

\section{Introduction}

Adrenocortical carcinoma (ACC) is a rare, highly aggressive endocrine malignancy derived from cells of the adrenal cortex and is often accompanied by unfavorable prognosis $(1,2)$. Despite significant advances in the last decade, its pathogenesis is only incompletely understood and overall therapeutic means are unsatisfactory (3). Therefore, it is urgent to find new treatment targets for ACC.

$\omega-3(n-3)$ and $\omega-6(n-6)$ polyunsaturated fatty acids (PUFAs) are essential fatty acids necessary for human health. Epidemiological studies suggest that diet with a high n-6/n-3 ratio contributes to cardiovascular disease, inflammation and cancer (4-6), while consumption of more fish or fish oil with a high $n-3 / n-6$ ratio could reduce the risk of colon, prostate and breast cancers $(7,8)$. Laboratory and animal studies have shown that n-3 PUFAs inhibit the growth of various types of cancers both in vitro and in vivo and affect emotions through the hypothalamic-pituitary-adrenal (HPA) axis $(9,10)$, as well as catecholamine handling through adrenal chromaffin cells (11), suggesting that n-3 PUFAs may affect adrenal structure and function. However, the effect of n-3 PUFAs on ACC is not known.

Mammalian target of rapamycin (mTOR) is a highly conserved Ser/Thr kinase composed of two functionally distinct signaling complexes, mTOR complex 1 (mTORC1) and complex 2 (mTORC2) (12). mTOR is a key molecule for controlling cell growth, proliferation, survival and metabolism $(12,13)$. Upregulation of mTOR signaling has been found in many cancers and is thought to play important roles in carcinogenesis and tumor progression (14-16). The mTOR pathway is also activated in a subset of adrenal tumors, but its role remains unclear.

We previously developed a transgenic mouse model that expresses fat-1, a desaturase that converts n-6 PUFAs to n-3 PUFAs and have applied it to study the effects of n-3 PUFAs on breast cancer $(17,18)$. In the present study, we investigated the potential effects of exogenous and endogenous n-3 PUFAs 
on the growth of ACC cell lines and tumor xenografts and explored the possible signaling mechanisms.

\section{Materials and methods}

Materials. All cell culture reagents were obtained from Gibco-BRL Life Technologies (Grand Island, NY, USA). Arachidonic acid (AA) and docosahexenoic acid (DHA) were obtained from Cayman Chemical (Ann Arbor, MI, USA) and prepared as dimethyl sulfoxide (DMSO) stock solutions following the manufacturer's instructions. Insulin, primary antibody against $\beta$-actin and HRP-conjugated anti-mouse and anti-rabbit IgG were from Sigma (St. Louis, MO, USA). Primary antibodies against phospho-S6 (S235/236) were purchased from Cell Signaling Technology (Danvers, MA, USA). Anti-S6, phospho-Akt (S473) and Akt antibodies were obtained from Santa Cruz Biotechnology, Inc. (Santa Cruz, CA, USA). pST180 control vector and pST180-fat-1 vector were chemically synthesized by GenePharma Co., Ltd. (Shanghai, China).

Cell lines and culture conditions. The human ACC cell lines SW13 and H295R were used in the present study. SW13 was preserved in our laboratory in L-15 supplemented with $10 \%$ fetal bovine serum (FBS) (Invitrogen Corp., Grand Island, NY, USA) and cultured in a $37^{\circ} \mathrm{C}$ humidified atmosphere containing $100 \%$ air and no $\mathrm{CO}_{2}$. The $\mathrm{H} 295 \mathrm{R}$ cell line was obtained from the Cell Center of the Chinese Academy of Sciences, maintained in Dulbecco's modified Eagle's medium (DMEM) supplemented with $15 \% \mathrm{FBS}$ and cultured in a $37^{\circ} \mathrm{C}$ humidified atmosphere containing $95 \%$ air and $5 \% \mathrm{CO}_{2}$. Cells were trypsinized upon confluency and propagated to passage 2 before being subcultured in 6-, 12- or 96-well plates for the experiments.

Cell proliferation assay. Cells were seeded at $2 \times 10^{3}$ cells/well in 96-well plates. After being cultured in medium containing $10 \% \mathrm{FBS}$ for $24 \mathrm{~h}$, the cells were treated with DHA at different concentrations (10-80 $\mu \mathrm{M})$ for $72 \mathrm{~h}$ with refreshing every $24 \mathrm{~h}$. Cell viability was then assessed using Cell Counting Kit-8 (CCK-8) (cat no. KGA317; KeyGen Biotech, Nanjing, China) following the manufacturer's instructions. The viability of the SW13 cells was similarly assessed at $48 \mathrm{~h}$ after transfection with the pST180-fat- 1 or control vector.

Colony formation assay. Colony formation assays were performed as previously described (19). Briefly, the cells were seeded in 6-well plates in triplicate at a density of 200 cells/well containing $2 \mathrm{ml}$ medium with 10\% FBS. Twenty-four hours later, the cells were cultured in fresh medium containing 5\% FBS alone as control or with DHA at different concentrations $\left(5\right.$ and $20 \mu \mathrm{M}$ ) for 14 days at $37^{\circ} \mathrm{C}$. The formed colonies were stained for 15 min with a solution containing $0.5 \%$ crystal violet and $25 \%$ methanol, followed by washing with water to remove excessive dye. The colony numbers were counted by Quantity One ${ }^{\circledR}$ 1-D analysis software (Bio-Rad Laboratories Inc., Hercules, CA, USA).

Flow cytometric analysis. Flow cytometric analysis was performed as previously described to determine the effects of DHA on the cell cycle (19). Briefly, SW13 cells grown in 6 -well plates $\left(2 \times 10^{5}\right.$ cells/well) were starved for $24 \mathrm{~h}$ in basal medium to synchronize at the G1/S boundary, and were then incubated in medium containing 10\% FBS alone or with $20 \mu \mathrm{M}$ DHA. Twenty-four hours later, the cells were harvested by trypsinization and fixed with $70 \%$ ethanol. Cell numbers were assessed following the manufacturer's protocol (KeyGen Biotech), and cell cycle distribution was analyzed by flow cytometry (FACSCalibur; BD Biosciences, Bedford, MA, USA). DNA histograms were plotted using FCS Express software, and the percentage of cells at the G0/G1, S and $\mathrm{G} 2 / \mathrm{M}$ stages were calculated.

Apoptosis assessment of cells and tissues. The Annexin V-FITC apoptosis detection kit was used for the apoptosis assay (KeyGen Biotech). In brief, SW13 cells $\left(1 \times 10^{6}\right.$ cells $\left./ \mathrm{ml}\right)$ were treated with $25 \mu \mathrm{M}$ DHA for $12 \mathrm{~h}$, harvested by trypsinization, washed twice with phosphate-buffered saline (PBS), and resuspended in $500 \mu \mathrm{l}$ of binding buffer. The cells were then incubated with $5 \mu \mathrm{l}$ of Annexin V-FITC and $5 \mu \mathrm{l}$ of propidium iodide (PI) for $10 \mathrm{~min}$ at room temperature in the dark and evaluated immediately by flow cytometry (FACSCalibur).

Apoptotic cell death in paraffin-embedded tumor tissue sections was examined using the DeadEnd ${ }^{\mathrm{TM}}$ Colorimetric TUNEL System (Promega, Madison, WI, USA) according to the manufacturer's protocol. Apoptotic cells were identified as dark brown nuclei under a light microscope. The number of apoptotic cells was counted in five random fields (magnification, $\mathrm{x} 400)$ in a blinded manner.

Western blot analysis. Protein expression levels were determined by western blot analysis as previously described (20). Briefly, after treatment for the indicated time, cells were lysed immediately in Laemmli buffer $(62.5 \mathrm{mM}$ Tris- $\mathrm{HCl} \mathrm{pH} 6.8$, $2 \%$ SDS, $10 \%$ glycerol, $50 \mathrm{mM}$ dithiothreitol, $0.01 \%$ bromophenol blue) for $5 \mathrm{~min}$ at $95^{\circ} \mathrm{C}$. The cell lysates were resolved by SDS/PAGE and transferred electrophoretically to nitrocellulose membranes (Bio-Rad). The membranes were incubated with specific antibodies and immunoreactive proteins were revealed using an enhanced chemiluminescence (ECL) kit (Santa Cruz Biotechnology, Inc.).

Xenografts in nude mice. Four-week-old female nude mice were purchased from the Experimental Animal Center of Southern Medical University (Guangzhou, China) and housed in a specific pathogen-free facility in accordance with guidelines established by the Committee on Animal Research of the Southern Medical University. SW13 cells at early passages were harvested, prepared as $3 \times 10^{6}$ cells $/ 100 \mu 1$ PBS suspension and implanted subcutaneously into the right flanks of each mouse. The animals were then randomly assigned into two groups with 6 mice each and fed with a normal (low) or high n-3 PUFA diet (21). Bidimensional tumor measurements were taken every 2 days. Tumor volume (V) was calculated as: $\mathrm{V}=0.52$ (length $\mathrm{x}$ width ${ }^{2}$ ). On day 29 after SW13 cells were implanted, all animals were sacrificed and the tumors were collected and weighed. The volume of the excised tumors was calculated as: $\mathrm{V}=0.52$ (length $\mathrm{x}$ width $\mathrm{x}$ depth). Various tumor specimens were minced and lysed for western blot analysis, while others were fixed in $4 \%$ formaldehyde, embedded in 
Table I. Primer sequences for PCR amplification.

\begin{tabular}{lll}
\hline Gene & Forward $\left(5^{\prime} \rightarrow 3^{\prime}\right)$ & \multicolumn{1}{c}{ Reverse $\left(5^{\prime} \rightarrow 3^{\prime}\right)$} \\
\hline fat -1 & GGACCTGGTGAAGAGCATCCG & GCCGTCGCAGAAGCCAAAC \\
SCID & GGAAAAGAATTGGTATCCAC & AGTTATAACAGCTGGGTTGGC
\end{tabular}

A

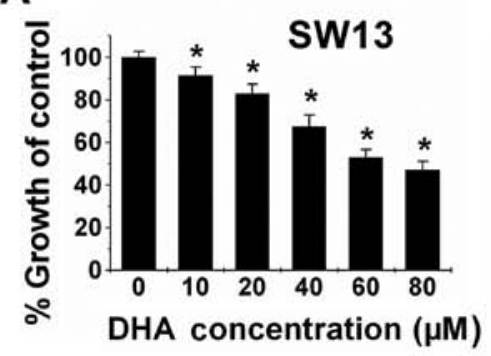

B

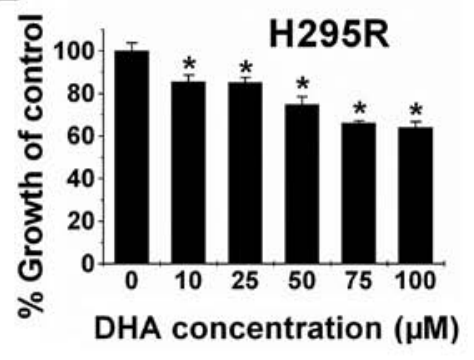

C

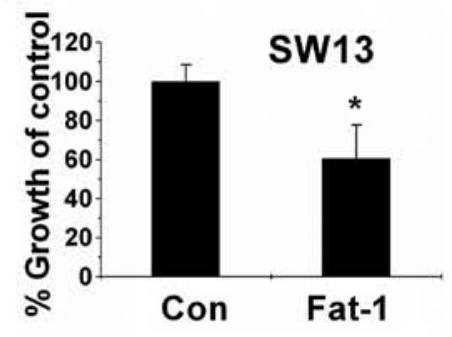

D

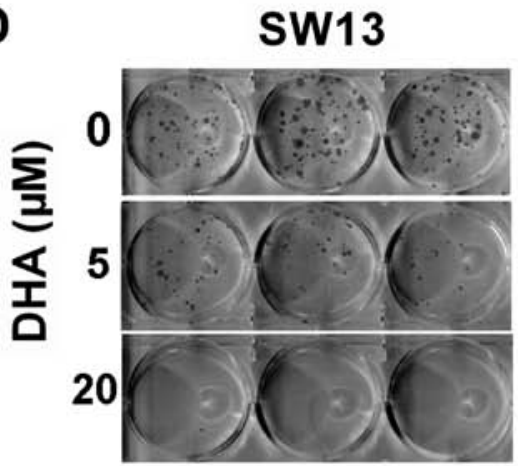

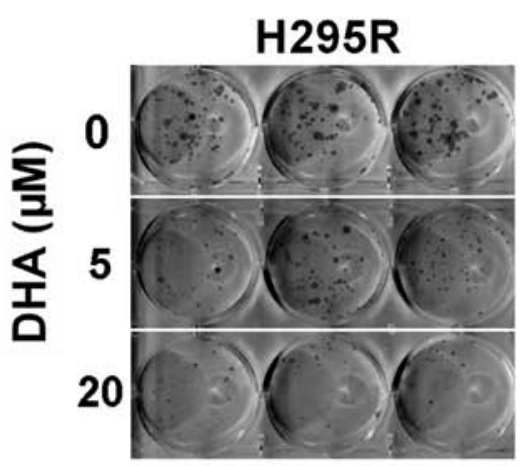

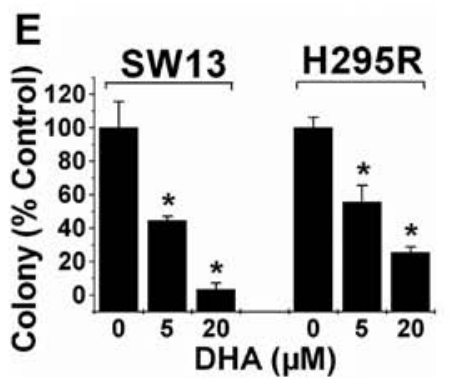

Figure 1. Inhibitory effect of n-3 PUFAs on the proliferation of ACC cells. Viability of the (A) SW13 and (B) H295R cells as assessed using Cell Counting Kit-8. The cells were plated in 96-well plates and treated with serial concentrations of DHA (0-100 $\mu \mathrm{M})$ for $48 \mathrm{~h}$, with DHA replaced every $24 \mathrm{~h}$. Bars indicate mean \pm SD. Statistics were performed by one-way ANOVA with application of LSD post test. ${ }^{*} \mathrm{P}<0.05$, compared with the group without DHA. (C) Viability of the SW13 cells at $48 \mathrm{~h}$ post transfection with the pST 180-fat-1 or control vector, respectively. Bars indicate mean \pm SD. ${ }^{*} \mathrm{P}<0.05$, compared with the control by Student's t-test. (D) ACC cells (SW13 and H295R) grown in 6-well plates were incubated with different concentrations $(0,5$ and $20 \mu \mathrm{M})$ of DHA for 2 weeks. The images of 6-well plates with colonies were captured by a digital camera on day 14. (E) The numbers of the cell colonies were obtained and counted by Quantity One 1-D gel quantity software as described in Materials and methods. Bars indicate mean \pm SD. Statistics were performed by one-way ANOVA with application of LSD post test. "P $<0.05$, compared with the group without DHA.

paraffin and cut in $4-\mu \mathrm{m}$ sections for immunohistochemical analysis.

Xenografts in severe combined immune deficiency (SCID) and fat-1-SCID mice. Homozygous SCID mice (Jax no. 001131) (BALB/c background) were bred with fat-1 transgenic mice (originally on the C57BL/6 background) produced previously to generate fat-1-SCID double-hybrid mice. These mice were backcrossed with BALB/c mice for 10 generations. Female littermates lacking the fat- 1 transgenic gene were used as controls. DNA extractions from the tail tips of offspring were subjected to PCR for genotyping in accordance with the protocol on the Jackson Laboratory webpage, using primers listed in Table I. Four-week-old female homozygous SCID mice $(n=6)$ with or without the fat- 1 gene were injected with $100 \mu \mathrm{l}\left(1 \times 10^{5}\right)$ of the SW13 cell suspension and were subjected to bidimensional tumor measurement and tumor sample analysis as described above.
Immunohistochemistry. Tumor allografts were removed. After being weighed, they were immediately fixed in $2.5 \%$ glutaraldehyde-polyoxymethylene solution and processed as paraffin sections using a standard method. Three sequential sections $(5 \mu \mathrm{m})$ of each sample were used for hematoxylin and eosin (H\&E) staining, immunohistochemical (IHC) detection of phospho-S6 (S235/S236) (1:300; Cell Signaling Technology) and IHC detection of phospho-Akt (S473) (1:100; Santa Cruz Biotechnology, Inc.), respectively. Cells with yellow brown cytoplasm or nuclei were considered positive. The percentage of positive cells was calculated after counting 1,000 cells at higher magnification (x400) according to the following formula: Percentage of positive cells $=$ (number of positive cells/number of total cells) x $100 \%$.

Statistical analysis. Statistical analyses were performed using SPSS (version 13.0). Data are presented as mean \pm SD of at least three independent experiments. Differences between two 
A

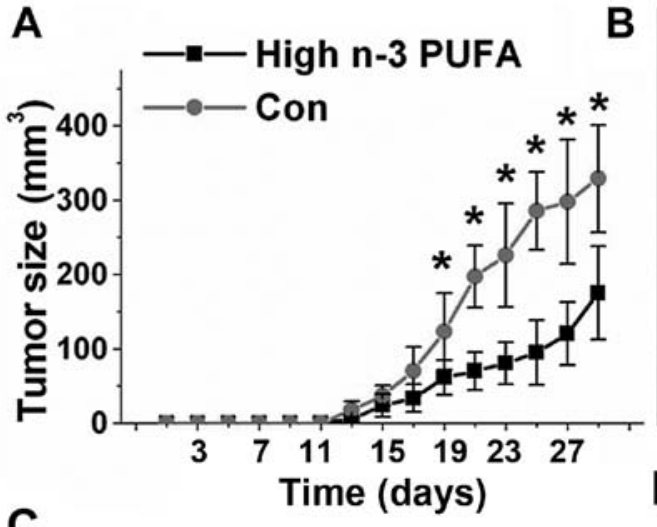

C

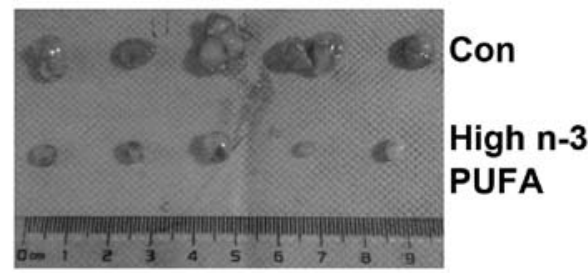

B
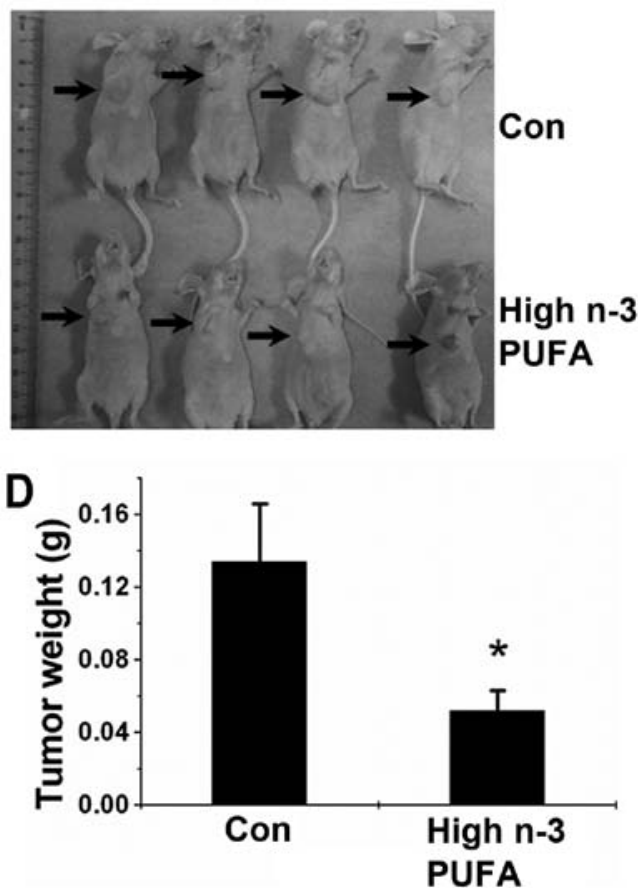

Figure 2. Dietary n-3 PUFAs inhibit the growth of ACC tumor xenografts. Xenografts were generated by subcutaneously implanting $3 \times 10^{6}$ SW13 cells into the flanks of nude mice, which were then randomized into two groups (6 mice/group) and subjected to a normal (low) or high n-3 PUFA diet. Tumor length (R2) and width (R1) were measured every 2 days, and tumor volume was estimated as $V=0.52 R_{1}{ }^{2} R_{2}$. On day 29 , the tumors were removed and weighed. (A) Twenty-nine day tumor growth curve in xenograft model mice. Bars indicate mean \pm SD. * $\mathrm{P}<0.05$, compared with the respective controls by Student's t-test. (B) Images of mice in the control and treated groups. The arrows point to the SW13 allografts. (C) Images of the excised tumors of mice in the control and treated groups. (D) A comparison of average tumor weight on the final day between the two groups. Bars indicate mean \pm SD. ${ }^{*} \mathrm{P}<0.05$, compared with the control by Student's t-test.

groups were analyzed using the Student's t-test, and differences among more than two groups were analyzed using one-way ANOVA and post hoc LSD tests. A $\mathrm{P}<0.05$ was considered as statistically significant. In the case of western blot analysis, one representative set of data is shown.

\section{Results}

n-3 PUFAs inhibit ACC cell proliferation and colony formation and induce G0/G1 cell cycle arrest. To investigate the potential protective role of n-3 PUFAs against ACC, we examined the effect of DHA on the proliferation of ACC cell lines. We treated SW13 and H295R cells with various concentrations $(0-100 \mu \mathrm{M})$ of DHA and assessed the effects after $48 \mathrm{~h}$ of treatment. As shown in Fig. 1A and B, DHA significantly inhibited the proliferation of both ACC cell lines in a dose-dependent manner. Transgenic expression of fat-1 is capable of converting n-6 PUFAs to n-3 PUFAs, leading to an increase in the amount of n-3 PUFAs and a decrease in the n-6/n-3 ratio. In addition, SW13 cells were transfected with the fat 1 or control vector, and cell viability was assessed $48 \mathrm{~h}$ later. As expected, the proliferation rate of the fat-1-expressing cells was significantly decreased by almost $40 \%$ compared with that noted in the control cells (Fig. 1C). Together, these findings demonstrate that both exogenous and endogenous n-3 PUFAs are effective to inhibit ACC cell proliferation.

We next examined the effects of DHA on cell colony formation of ACC cells. Our results showed that DHA prevented colony formation of the ACC cells in a dose- dependent manner. When used at $5 \mu \mathrm{M}$ DHA, DHA inhibited the cell colony formation of the SW13 and H295R cells by 60 and $50 \%$, respectively. Additionally, $20 \mu \mathrm{M}$ DHA decreased the colony formation of the SW13 and H295R cells by 97 and $75 \%$, respectively, compared with the control (Fig. 1D and E).

We also examined the effects of DHA on cell cycle progression by flow cytometric analysis. The SW13 cells were serum-starved for $24 \mathrm{~h}$ to arrest cells at the G0/G1 phase. After refreshing the medium, FBS was added to the arrested cells for $48 \mathrm{~h}$ in the presence or absence of DHA. DHA-treated cells were arrested at G0/G1, whereas FBS-treated cells progressed to the $\mathrm{S}$ phase (data not shown). However, this effect was not significant.

Dietary n-3 PUFAs inhibit the growth of ACC xenografts in nude mice. After showing the inhibitory effects of n-3 PUFAs on the proliferation of ACC cells, we examined the ability of n-3 PUFAs to inhibit tumor growth in vivo. We established an ACC xenograft model by subcutaneously implanting SW13 cells into nude mice. The animals were administered a normal (low) or high n-3 PUFA diet. As shown in Fig. 2, a high n-3 PUFA diet efficiently prevented tumor growth and reduced the average tumor weight and volume. Fatty acid composition analysis identified a significantly increased ratio of $n-3 / n-6$ PUFAs in the tumors of mice fed a high n-3 PUFA diet when compared with that of mice fed a normal diet (Table II).

Endogenously produced n-3 PUFAs inhibit the growth of ACC xenografts in SCID mice. Transgenic expression of fat-1 
Table II. Fatty acid composition in the nude mouse tumors.

Type of fatty acids

n-3 PUFA diet

(mol \% of total fatty acid)

\begin{tabular}{rc}
\hline \multicolumn{1}{c}{ Normal } & High \\
$0.25 \pm 0.12$ & $0.44 \pm 0.08$ \\
$0.13 \pm 0.02$ & $0.96 \pm 0.05$ \\
$0.56 \pm 0.11$ & $1.23 \pm 0.14$ \\
$2.15 \pm 0.38$ & $4.37 \pm 0.33$ \\
$3.08 \pm 0.33$ & $7.00 \pm 0.40^{\mathrm{a}}$ \\
$19.24 \pm 3.12$ & $17.32 \pm 2.17$ \\
$1.75 \pm 0.37$ & $1.22 \pm 0.18$ \\
$20.99 \pm 2.92$ & $18.55 \pm 2.08$ \\
$0.15 \pm 0.02$ & $0.38 \pm 0.04^{\mathrm{a}}$
\end{tabular}

C18:3 n-3, $\alpha$-linoleic acid

C20:5, n-3, eicosapentaenoic acid (EPA)

$0.13 \pm 0.02$

$0.44 \pm 0.08$

C22:5, n-3, docosapentaenoic acid (DPA)

$0.56 \pm 0.11$

$1.23 \pm 0.14$

C22:6, n-3, docosahexaenoic acid (DHA)

$2.15 \pm 0.38$

$4.37 \pm 0.33$

$\mathrm{n}-3$, total

$19.24 \pm 3.12$

$17.32 \pm 2.17$

C18:2, n-6, linoleic acid

$1.75 \pm 0.37$

n-6, total

$0.15 \pm 0.02$

$0.38 \pm 0.04^{\mathrm{a}}$

Values of n-3 and n-6 in mammary tumors $(n=6)$ were measured by gas chromatography-mass spectroscopy. Data indicate mean \pm SD. ${ }^{a}<0.01$, compared with a normal n-3 PUFA diet. PUFAs, polyunsaturated fatty acids.
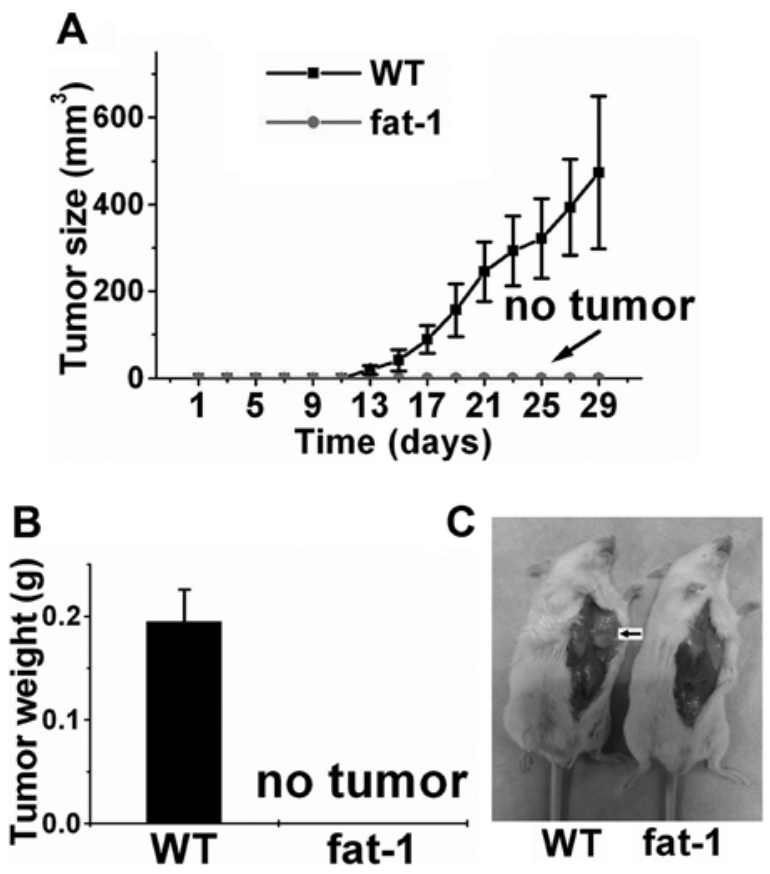

Figure 3. Endogenously produced n-3 PUFAs inhibit ACC growth in a xenograft model of fat-1-SCID mice. SCID mice or fat-1-SCID were implanted with SW13 cells as described in Materials and methods. (A) Twenty-nine-day tumor growth curve in the SCID mouse model. (B) Comparison of tumor mass on the final day between the two indicated groups in the SCID mouse models. Bars indicate mean \pm SD. (C) Representative images of tumors in situ after exposure of the abdominal inner skin.

is capable of converting n-6 PUFAs to n-3 PUFAs, leading to an increase in n-3 PUFAs and a decrease in the $n-6 / n-3$ ratio, and allows us to perform well-controlled studies in the absence of restricted diets. For this purpose, we previously established fat 1 transgenic SCID mice and confirmed that the ratio of n-3/n-6 PUFAs in the fat- 1 transgenic SCID mice is significantly increased compared with this ratio in the wild-type (WT) SCID mice lacking fat-1 expression (22). Notably, although xenograft tumors with an average volume of $473 \mathrm{~mm}^{3}$ were observed within 4 weeks in the control SCID mice, we failed to observe tumor growth in any of the fat-1 SCID mice (Fig. 3), suggesting that ACC cells are unable to proliferative or survive in the presence of high levels of endogenously produced n-3 PUFAs in vivo.

n-3 PUFAs promote ACC cell apoptosis both in vitro and in vivo. We further examined whether n-3 PUFAs could induce ACC cell apoptosis in vitro and in xenografts. The results showed that DHA significantly increased the number of apoptotic cells in serum-starved SW13 cells but not in SW13 cells cultured with normal FBS (Fig. 4A and B), suggesting that DHA promotes ACC cell apoptosis in vitro. The effect of n-3 PUFAs on apoptosis was further examined in xenograft models. The results revealed that administration of n-3 PUFAs promoted xenografted ACC tumor apoptosis as manifested by the increased numbers of TUNEL-positive tumor cells in mice fed a high n-3 PUFA diet (Fig. 4C and D). We conclude that n-3 PUFAs promote ACC cell apoptosis both in vitro and in vivo.

n-3 PUFAs inhibit mTORC1/2 signaling in ACC cell lines and a xenograft mouse model. To investigate the underlying mechanisms of the in vitro effect of n-3 PUFAs on mTORC1/2 signaling, we first examined whether DHA (exogenous n-3 PUFAs) inhibited mTORC1/2 in the ACC cells. In the SW13 cells, DHA rapidly and dose-dependently suppressed insulin-stimulated mTORC1-directed phosphorylation of S6 (S235/235) and mTORC2-directed phosphorylation of Akt (S473) (Fig. 5A). We next examined the role of endogenously produced $n-3$ PUFAs in mTORC1/2 signaling. In the SW13 cells transfected with fat-1 cDNA, phosphorylation of S6 (S235/235) and Akt (S473) was significantly reduced compared with the cells transfected with the control vector (Fig. 5B). These results suggest that both the mTORC1 and mTORC2 signaling pathways are targets of exogenous and endogenous n-3 PUFAs in ACC cells. 
A

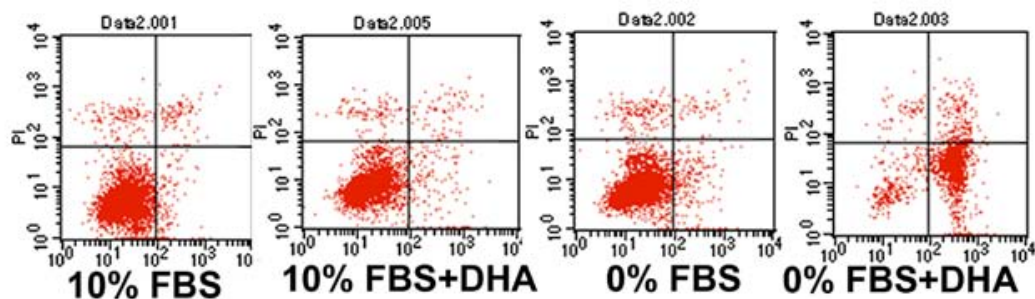

B

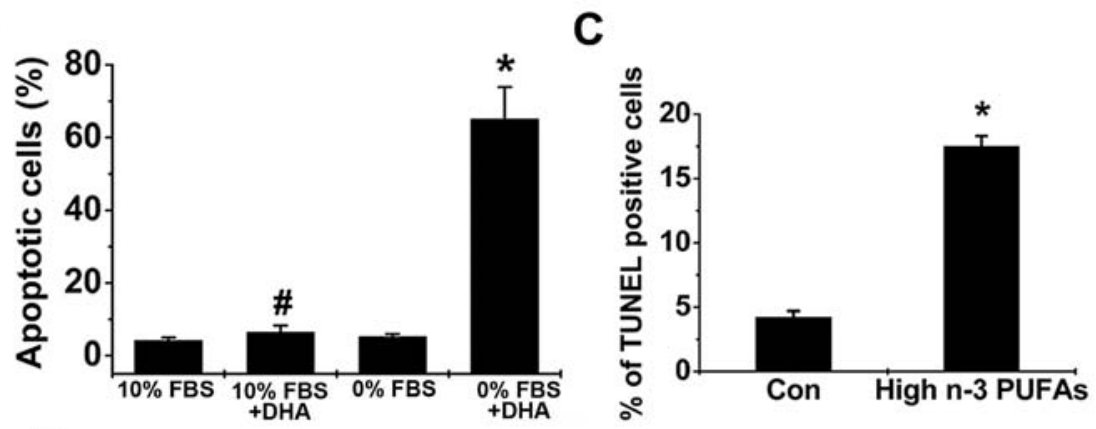

D
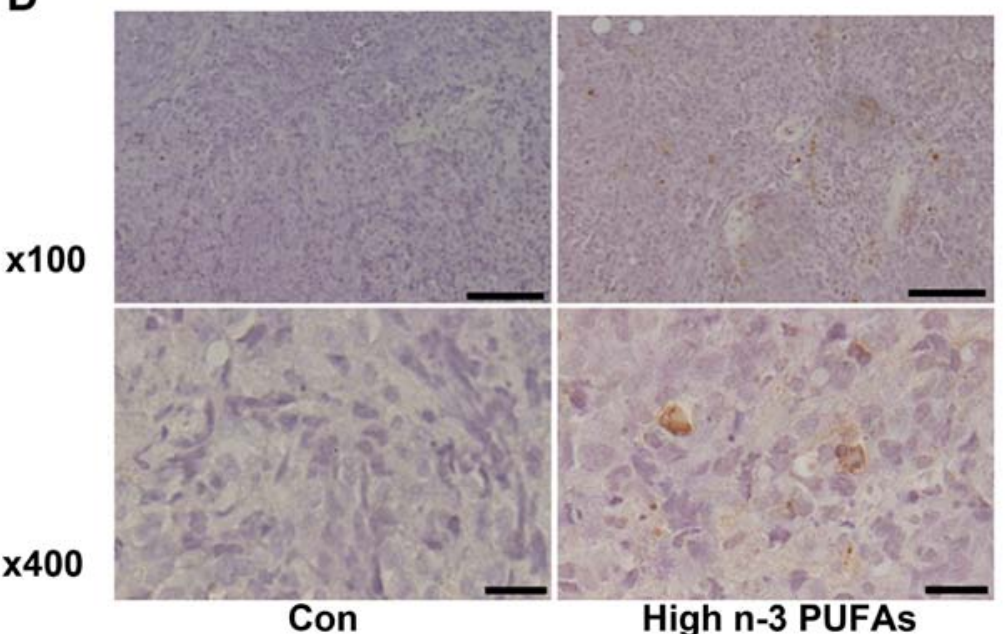

Figure 4. n-3 PUFAs promote ACC cell apoptosis in vitro and in vivo. (A) Shown are the representative images of flow cytometric analysis of SW13 cells cultured in medium with or without $10 \%$ FBS. Cells were treated with $25 \mu \mathrm{M}$ DHA or without DHA for $12 \mathrm{~h}$, stained with Annexin V-FITC and PI and immediately subjected to flow cytometry. (B) The percentage of apoptotic cells in each group. Bars indicate mean \pm SD. Statistics were performed by one-way ANOVA with application of LSD post test. "P<0.05 and " $\mathrm{P}>0.05$, compared with the respective controls. (C) Nude mice injected with a suspension of SW13 cells were administered a normal (Con) or high n-3 PUFA diet for 29 days and analyzed using DeadEnd ${ }^{\mathrm{TM}}$ Colorimetric TUNEL System. The number of apoptotic cells in five random fields (magnification, $\mathrm{x} 400$ ) was counted in a blinded manner. Bars indicate mean $\pm \mathrm{SD}$. ${ }^{*} \mathrm{P}<0.05$, compared with the control by Student's t-test. (D) Representative images of tumor sections examined by TUNEL assay. Apoptotic cells were identified as dark brown nuclei under a fluorescence microscope (magnifications, x100 and x400).

We next determined whether n-3 PUFAs could suppress mTORC1/2 in vivo. The levels of mTORC1/2 signaling in the excised tumors of the different groups were examined by immunohistochemical staining and western blotting. As shown in Fig. 5C, a high n-3 PUFA diet decreased the percentage of p-S6 (S235/236) and p-Akt (S473)-positive cells. Western blot analysis also showed that a high n-3 PUFA diet resulted in a significant reduction in levels of phosphorylated S6 (S235/236) and Akt (S473) (Fig. 5D). These data suggest that mTORC1 and mTORC2 signaling are the targets of $\mathrm{n}-3$ PUFAs in vivo and suppression of $\mathrm{mTORC} 1 / 2$ signaling by $\mathrm{n}-3$ PUFAs may contribute to their inhibitory effects on ACC tumor growth.

n-6 PUFAs activate mTORC1 signaling in ACC cells and stimulate ACC cell proliferation. Several previous studies have shown that arachidonic acid (AA) and its metabolites stimulate the growth and proliferation of various tumor cells $(6,23-25)$, suggesting that $n-6$ PUFAs may have opposite effects on cancer when compared with n-3 PUFAs. However, whether AA has any effect on ACC cell proliferation has not been reported. As shown in Fig. 6B, AA dose-dependently stimulated SW13 cell proliferation from 0.1 to $5 \mu \mathrm{M}$, and increased mTORC1-directed phosphorylation of S6 (S235/235) (Fig. 6A) in the SW13 cells. To further explore whether mTOR signaling plays a role in AA-induced cell growth, we examined the effect of rapamycin, an mTORC1 inhibitor, on SW13 cell proliferation. As shown in Fig. 6C, rapamycin treatment significantly suppressed AA-stimulated growth, suggesting that $\mathrm{mTORC} 1$ is required for AA-enhanced SW13 cell proliferation. 
A

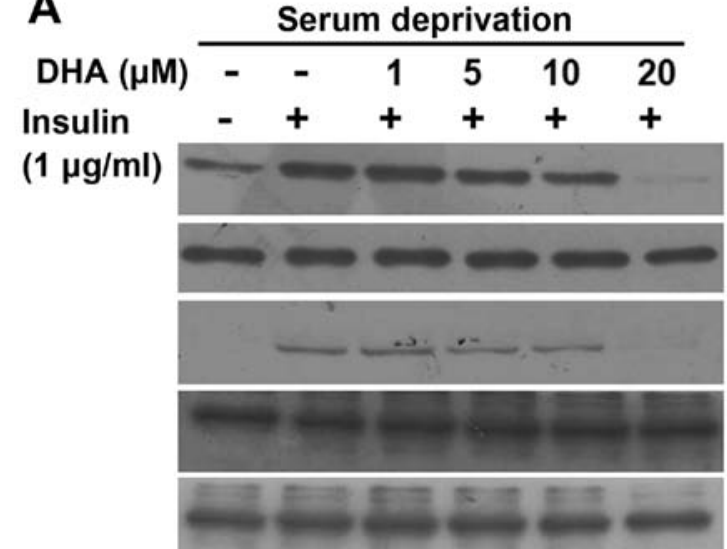

B

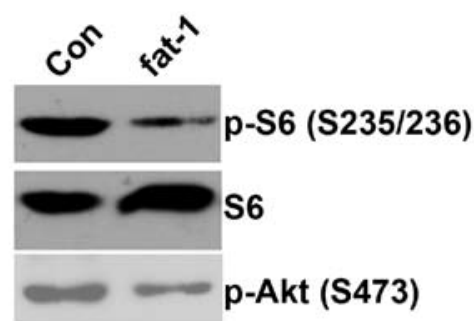

Akt
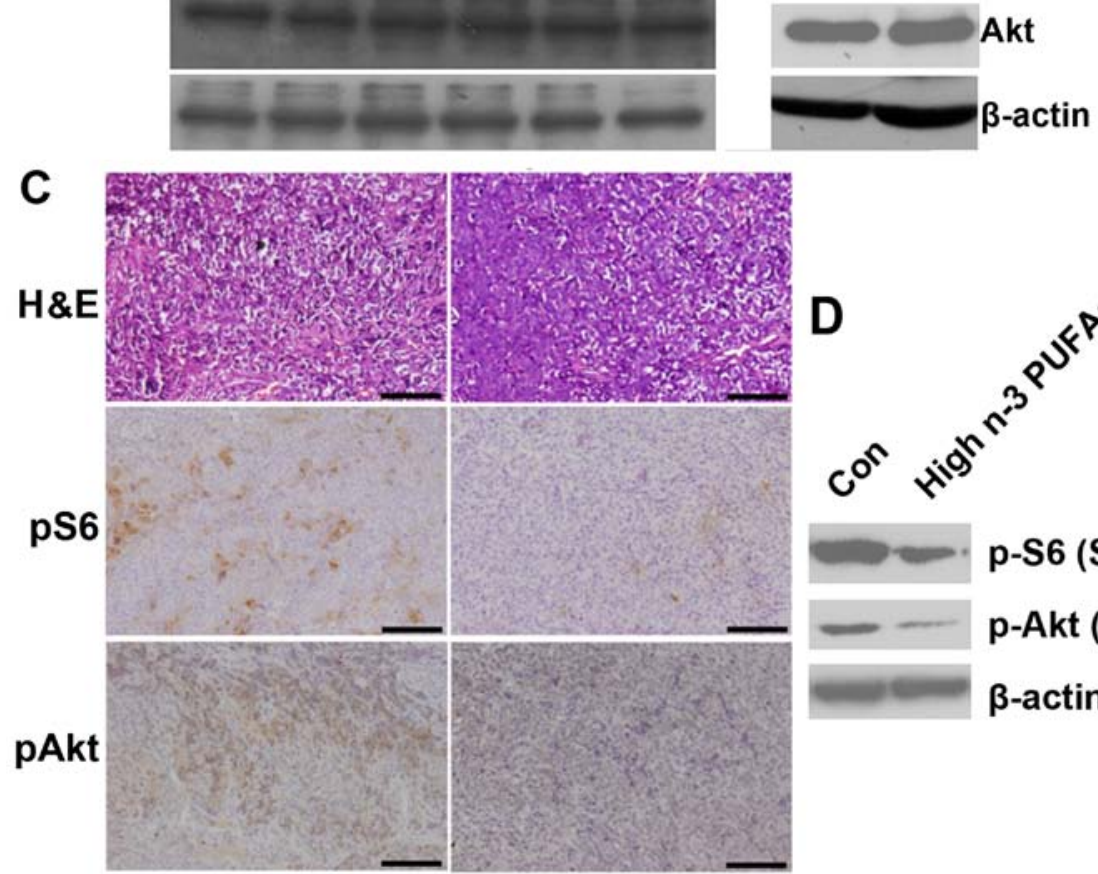

Con

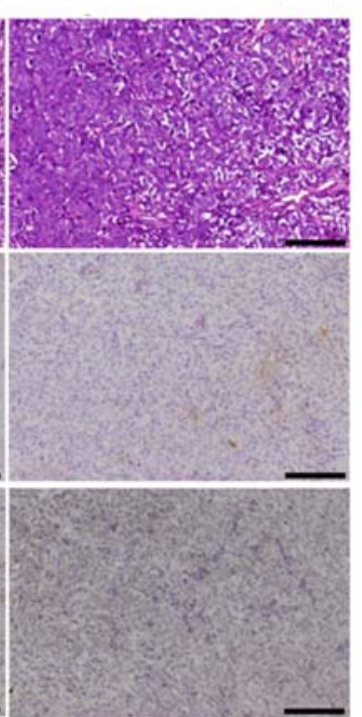

High n-3 PUFAs

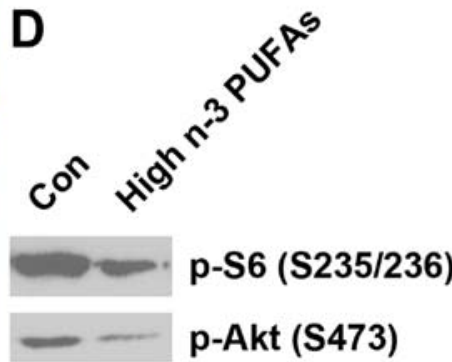

$\beta$-actin

Figure 5. n-3 PUFAs inhibit mTORC1/2 signaling pathways in vitro and in vivo. (A) SW13 cells were serum-starved for 18 h before treatment with the indicated concentrations of DHA for $30 \mathrm{~min}$, followed by insulin $(1 \mu \mathrm{g} / \mathrm{ml})$ stimulation for another $30 \mathrm{~min}$. (B) SW13 cells transfected with the pST180 empty vector (Con) or pST-180-fat-1 (fat-1) were serum-starved for $18 \mathrm{~h}$. The protein extract from each set was subjected to western blot analysis of mTORC1/2 signaling. (C) Nude mice injected with a suspension of SW13 cells were administered a normal (Con) or high n-3 PUFA diet for 29 days. mTORC1/2 signaling in ACC tumor tissues of each group was determined by immunohistochemistry and western blotting. Representative images of the tumor sections examined by immunohistochemical staining for p-S6 (S235/236) and p-Akt (S473). High n-3 PUFA diet treatment caused a lower percentage of p-S6- and p-Akt-positive cells. (D) Levels of p-S6 (S235/236) and p-Akt (S473) in tumors were determined by western blotting.

\section{Discussion}

The present study, for the first time, investigated the roles of n-3 PUFAs in the growth of ACC using in vitro cultured cells and an animal model. We found that DHA, a n-3 PUFA, exhibits strong anticancer activity in human ACC cells through a combination of multiple actions, including inhibition of cell proliferation and cell cycle progression and induction of cell apoptosis. The strong anticancer effect was also observed in vivo in tumor-bearing mice. All this evidence indicates that exogenous and endogenous n-3 PUFAs inhibit ACC cell growth both in vitro and in vivo.

Although increasing evidence from animal and in vitro studies indicate that n-3 PUFAs present in fatty fish and fish oils inhibit the carcinogenesis of many types of tumors, inconsistencies remain $(4,21)$. Several factors may account for these inconsistent results including: i) a wide variations in the amount and source of n-3 PUFAs consumed in each study; and ii) the ratio of $n-6$ to $n-3$, which may be more important than the absolute amount of n-3 PUFAs, as suggested by animal and human studies (6). Transgenic expression of fat-1 enables the host to produce n-3 PUFAs endogenously while concomitantly reducing the levels of n-6 PUFAs $(17,21)$. In addition, the fat- 1 transgenic mouse is capable of increasing n-3 content with a balanced n-6/n-3 PUFA ratio in all tissues and allows carefully controlled studies to be performed in the absence of restricted diets $(17,21)$. We found that ACC cells did not grow at all in fat- 1 transgenic SCID mice possibly since the transplanted cells the in fat- 1 transgenic SCID mice underwent growth inhibition and apoptosis after uptaking n-3 PUFAs from their immediate environment. Due to the lack of a reagent available to induce adrenal tumorigenesis and a genetically induced animal model with adrenal tumorigenesis, it was not possible for us to investigate whether n-3 PUFAs have any effect on adrenal carcinogenesis and progression. However, we can conclude from our results that exogenous 


\section{A Serum deprivation}
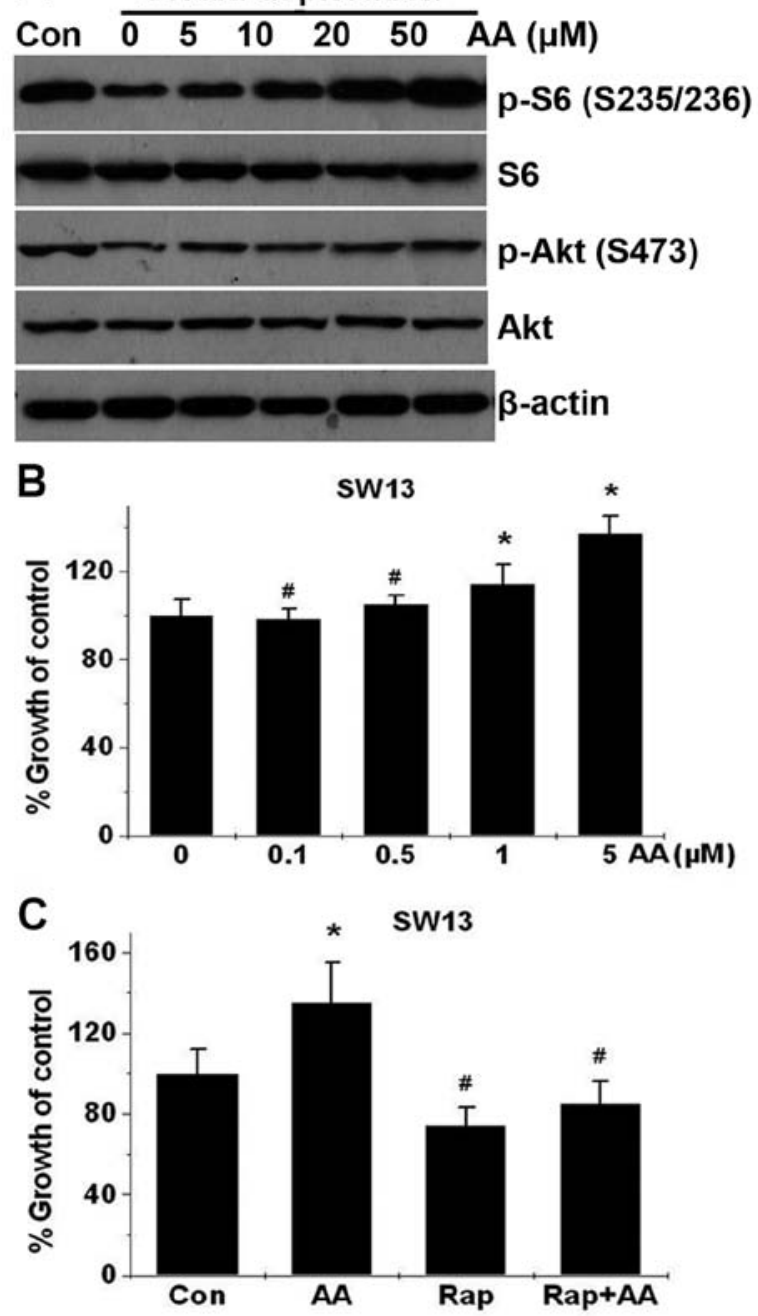

Figure 6. Arachidonic acid (AA) activates mTORC1 signaling and stimulates ACC cell proliferation. (A) Western blot analyses of phospho-S6 (S235/236) and S6 in the lysates of SW13 cells treated with $30 \mu \mathrm{M}$ AA for the indicated times after $16 \mathrm{~h}$ of serum deprivation. (B) Assessment of viability using Cell Counting Kit- 8 of SW13 cells treated with serial concentrations of AA $(0-5 \mu \mathrm{M})$ for $72 \mathrm{~h}$, with AA refreshed every $24 \mathrm{~h}$. Bars indicate mean $\pm \mathrm{SD}$ Statistics were performed by one-way ANOVA with application of LSD post test. ${ }^{*} \mathrm{P}<0.05$ and ${ }^{\#} \mathrm{P}>0.05$, compared with the control. (C) Assessment of viability using Cell Counting Kit-8 of SW13 cells treated with $5 \mu \mathrm{M}$ of AA alone or in combination with $100 \mathrm{nM}$ of rapamycin for $48 \mathrm{~h}$. Bars indicate mean \pm SD. Statistics were performed by one-way ANOVA with application of LSD post test. ${ }^{*} \mathrm{P}<0.05$, compared with the control; ${ }^{\#} \mathrm{P}<0.05$, compared with the control and AA treatment.

and endogenous n-3 PUFAs can inhibit ACC growth both in vitro and in vivo.

Understanding n-3 PUFA-regulated signaling pathways in cancer may provide valuable information for assessing their potential value in both cancer prevention and treatment. We found that exogenous and endogenous n-3 PUFAs inhibited mTORC1 and mTORC2 signaling in the ACC cells and in the tissues of tumor-bearing mice. mTOR is a key molecule which integrates diverse signals including nutrients, growth factors, energy and stresses to control cell growth, proliferation, survival and metabolism $(12,13)$. mTOR signaling is upregulated in many types of cancers and plays key roles in the carcinogenesis and progression of these diseases, thus it is regarded as a key target for cancer therapy (14-16). mTORC1 inhibitors such as rapamycin and envirolumus have been used for the treatment of ACC in preclinical trials, but the results were not promising (26). The reason is that these drugs only block mTORC1 and have little effect on mTORC2, which has a feedback effect on mTORC1 signaling (20). The development of dual inhibitors of mTORC1 and mTORC2 may obviate this issue since they appear to exhibit a superior effect in tumor treatment than inhibitors of mTORC1 only (27). Our findings that n-3 PUFAs target both mTORC1 and mTORC2 pathways may explain their strong inhibitory effects on ACC in vitro and in vivo. The mechanisms by which n-3/n-6 PUFAs regulate mTORC1/2 remain to be identified.

We previously reported that AA-activated mTOR signaling plays a critical role in breast carcinogenesis and angiogenesis (25). Not surprisingly, in the present study, we also found that AA activated mTOR signaling in ACC cells and promoted ACC cell proliferation, which requires activation of the mTORC1 signaling pathway. Unfortunately, no animal model, which can mimic ACC development, can be used to explore the effects of n-6 PUFAs on adrenal carcinogenesis. Our results at least showed that n-6 PUFAs may be related to ACC growth and upregulation of mTOR signaling in ACC may be partly due to AA uptake. It has been reported that prostate or breast-specific knockout of TSC1 (which activates mTORC1) can lead to prostate or breast carcinogenesis $(28,29)$. Therefore, we can use the adrenal cortical-specific Cre mouse and TSC1loxp mouse to investigate whether adrenal mTORC1 activation could lead to adrenal hyperplasia, adenoma formation or ACC development and confirm the role of mTORC1 in adrenal carcinogenesis (30).

In conclusion, our results indicate that both endogenously synthesized and exogenously uptaken n-3 PUFAs inhibit ACC growth, while n-6 PUFAs have an opposite effect, the mechanisms of which may be mediated by mTORC1/2 signaling. However, further research is required to investigate the key role of mTORC1/2 signaling in adrenal carcinogenesis. In addition, future research must elucidate whether n-6 PUFA intake has any direct relationship with mTOR signaling activation in ACC.

\section{Acknowledgements}

The present study was supported by the National Natural Sciences Foundation of China (nos. 81302230 and 31371186), the China Postdoctoral Science Foundation (2013M542159), the Natural Science Foundation of Guangdong Province, China (2014A030313296), and the Guangdong Province Outstanding Young Teacher Training funds.

\section{References}

1. Ronchi CL, Kroiss M, Sbiera S, Deutschbein T and Fassnacht M: EJE prize 2014: Current and evolving treatment options in adrenocortical carcinoma: Where do we stand and where do we want to go? Eur J Endocrinol 171: R1-R11, 2014.

2. Wang W, Hu W, Zhang X, Wang B, Bin C and Huang H: Predictors of successful outcome after adrenalectomy for primary aldosteronism. Int Surg 97: 104-111, 2012.

3. Erickson LA, Rivera M and Zhang J: Adrenocortical carcinoma: Review and update. Adv Anat Pathol 21: 151-159, 2014.

4. Gerber M: $\Omega-3$ fatty acids and cancers: A systematic update review of epidemiological studies. Br J Nutr 107 (Suppl 2): S228-S239, 2012. 
5. Update on marine omega-3 fatty acids: Management of dyslipidemia and current omega-3 treatment options. Atherosclerosis 230 : 381-389, 2013

6. Kang JX and Liu A: The role of the tissue omega-6/omega-3 fatty acid ratio in regulating tumor angiogenesis. Cancer Metastasis Rev 32: 201-210, 2013.

7. Zhang F, Chen Y, Long J, Dong L, Wang Y and Chen Y: Effect of n-3 and n-6 polyunsaturated fatty acids on lipid metabolic genes and estrogen receptor expression in MCF-7 breast cancer cells. Clin Lab 61: 397-403, 2015.

8. de Roos B and Romagnolo DF: Proteomic approaches to predict bioavailability of fatty acids and their influence on cancer and chronic disease prevention. J Nutr 142: 1370S-1376S, 2012.

9. Abel S, Riedel S and Gelderblom WC: Dietary PUFA and cancer. Proc Nutr Soc 73: 361-367, 2014

10. Larrieu T, Hilal ML, Fourrier C, De Smedt-Peyrusse V, Sans N, Capuron L and Layé S: Nutritional omega-3 modulates neuronal morphology in the prefrontal cortex along with depression-related behaviour through corticosterone secretion. Transl Psychiatry 4: e437, 2014.

11. Gomes A, Correia G, Coelho M, Araújo JR, Pinho MJ, Teixeira AL, Medeiros R and Ribeiro L: Dietary unsaturated fatty acids differently affect catecholamine handling by adrenal chromaffin cells. J Nutr Biochem 26: 563-570, 2015.

12. Foster KG and Fingar DC: Mammalian target of rapamycin (mTOR): Conducting the cellular signaling symphony. J Biol Chem 285: 14071-14077, 2010.

13. Sengupta S, Peterson TR and Sabatini DM: Regulation of the mTOR complex 1 pathway by nutrients, growth factors, and stress. Mol Cell 40: 310-322, 2010.

14. Dazert E and Hall MN: mTOR signaling in disease. Curr Opin Cell Biol 23: 744-755, 2011.

15. Xu K, Liu P and Wei W: mTOR signaling in tumorigenesis. Biochim Biophys Acta 1846: 638-654, 2014.

16. Efeyan A and Sabatini DM: mTOR and cancer: Many loops in one pathway. Curr Opin Cell Biol 22: 169-176, 2010.

17. Kang JX: From fat to fat-1: a tale of omega-3 fatty acids. J Membr Biol 206: 165-172, 2005.

18. White PJ, Arita M, Taguchi R, Kang JX and Marette A: Transgenic restoration of long-chain $n-3$ fatty acids in insulin target tissues improves resolution capacity and alleviates obesitylinked inflammation and insulin resistance in high-fat-fed mice. Diabetes 59: 3066-3073, 2010.

19. Liu J, Li M, Song B, Jia C, Zhang L, Bai X and Hu W: Metformin inhibits renal cell carcinoma in vitro and in vivo xenograft. Urol Oncol 31: 264-270, 2013
20. Li M, Zhao L, Liu J, Liu A, Jia C, Ma D, Jiang Y and Bai X: Multi-mechanisms are involved in reactive oxygen species regulation of mTORC1 signaling. Cell Signal 22: 1469-1476, 2010.

21. Chen Z, Zhang Y, Jia C, Wang Y, Lai P, Zhou X, Wang Y, Song Q, Lin J, Ren Z, et al: mTORC1/2 targeted by $n-3$ polyunsaturated fatty acids in the prevention of mammary tumorigenesis and tumor progression. Oncogene 33: 4548-4557, 2014.

22. Zheng H, Tang H, Liu M, He M, Lai P, Dong H, Lin J, Jia C, Zhong M, Dai Y, et al: Inhibition of endometrial cancer by $\mathrm{n}-3$ polyunsaturated fatty acids in preclinical models. Cancer Prev Res 7: 824-834, 2014.

23. Kiyabu GY, Inoue M, Saito E, Abe SK, Sawada N, Ishihara J, Iwasaki M, Yamaji T, Shimazu T, Sasazuki S, et al; JPHC Study Group: Fish, n-3 polyunsaturated fatty acids and n- 6 polyunsaturated fatty acids intake and breast cancer risk: The Japan Public Health Center-based prospective study. Int J Cancer 137: 2915-2926, 2015.

24. Thomasz L, Oglio R, Rossich L, Villamar S, Perona M, Salvarredi L, Dagrosa A, Pisarev MA and Juvenal GJ: 6 Iodo- $\delta$ lactone: A derivative of arachidonic acid with antitumor effects in HT-29 colon cancer cells. Prostaglandins Leukot Essent Fatty Acids 88: 273-280, 2013.

25. Wen ZH, Su YC, Lai PL, Zhang Y, Xu YF, Zhao A, Yao GY, Jia CH, Lin J, Xu S, et al: Critical role of arachidonic acid-activated mTOR signaling in breast carcinogenesis and angiogenesis. Oncogene 32: 160-170, 2013.

26. Fraenkel M, Gueorguiev M, Barak D, Salmon A, Grossman AB and Gross DJ: Everolimus therapy for progressive adrenocortical cancer. Endocrine 44: 187-192, 2013

27. Zhou HY and Huang SL: Current development of the second generation of mTOR inhibitors as anticancer agents. Chin J Cancer 31: 8-18, 2012.

28. Hsu JL, Liu SP, Lee CC, Hsu LC, Ho YF, Huang HS and Guh JH: A unique amidoanthraquinone derivative displays antiproliferative activity against human hormone-refractory metastatic prostate cancers through activation of LKB1-AMPK-mTOR signaling pathway. Naunyn Schmiedebergs Arch Pharmacol 387: 979-990, 2014.

29. Pande M, Bondy ML, Do KA, Sahin AA, Ying J, Mills GB, Thompson PA and Brewster AM: Association between germline single nucleotide polymorphisms in the PI3K-AKT-mTOR pathway, obesity, and breast cancer disease-free survival. Breast Cancer Res Treat 147: 381-387, 2014.

30. Chen Z, Dong H, Jia C, Song Q, Chen J, Zhang Y, Lai P, Fan X, Zhou X, Liu M, et al: Activation of mTORC1 in collecting ducts causes hyperkalemia. J Am Soc Nephrol 25: 534-545, 2014. 